\title{
Sociedad española y Medicina del Trabajo del siglo XXI
}

Spanish society and Occupational Medicine in the XXI century

María Castellano Arroyo

Resumen

En este artículo se hace un repaso por los cambios ocurridos en la sociedad española desde la perspectiva demográfica, los movimientos migratorios, los modelos familiares, la cultura y la educación, la situación económica o la tecnología. Se analizan las características de la población activa, desempleada y jubilada. Desde esta perspectiva, se analizan los objetivos de la Medicina del Trabajo y su oportunidad de ofrecer aportaciones imaginativas en la sociedad del futuro, progresivamente más envejecida. La investigación, la promoción de la salud y la formación en hábitos saludables desde la edad laboral juvenil, la mejor integración de la población trabajadora, etc., proporcionará una mejor salud no solo en la época laboral, sino mucho más allá cuando se haya producido la jubilación.

\section{Abstract}

In this paper, we review the changes in Spanish society from a demographic perspective: migration, family patterns, culture and education, economic status or technology.

We analyze the characteristics of the labor force, unemployed and retired. From this perspective we analyze the aims of Occupational Medicine and its opportunity to offer imaginative contributions to our future society, progressively aging.

Research, health promotion and training in healthy habits since early labor age will provide a better health, not only in labor time, but beyond, until retirement. 


\section{INTRODUCCIÓN}

La gran aportación de Bernardino Ramazzini a la Medicina estuvo en su sagaz observación sobre los efectos que determinados trabajos tenían sobre la salud de quienes los realizaban ${ }^{1,2}$; de ahí derivó su propuesta de añadir, al interrogatorio hipocrático la cuarta pregunta: ¿En qué trabaja?; con ello se cerraba el círculo de respuestas al: ¿Qué le pasa?, ¿desde cuándo?, ¿a qué lo atribuye? En la Medicina tecnificada de hoy, el médico se olvida, con demasiada frecuencia, de orientar la anamnesis sobre estos cuatro pilares cuya información permite pasos sucesivos en el diagnóstico y el tratamiento progresivamente personalizados y desde la perspectiva integral de la persona que, también incluye su actividad laboral.

El profesor Reverte, para cumplir mejor el encargo recibido de escribir un artículo sobre la obra de Ramazzini, se desplazó a Italia y recorrió los lugares en los que éste desarrolló su obra, desde Carpi donde había nacido el 5 de noviembre de 1633, pasando por Parma, donde se doctoró en Filosofía, grado que completó con los estudios de Medicina a la que se dedicó toda su vida como docente y como médico, ejerciendo unos meses en Carpi y seguidamente en Módena desde 1673 hasta 1700, pasando después a Padova donde falleció. El artículo mencionado aporta datos muy detallados, y recogidos de los archivos originales, sobre la obra del padre de la Medicina del Trabajo, basada en lo evidente y estrechamente ligada a la situación social y económica de su tiempo ${ }^{3}$.

En el mundo, el proceso evolutivo se produce como consecuencia de la interrelación entre los cambios que van afectando a la sociedad, en todos sus órdenes (social, cultural, económico, científico, laboral, familiar, etc.), unos van influyendo sobre otros en un constante cambio que se contagia entre países y continentes convirtiéndose en un proceso, como decimos hoy, global. En el centro de todo ello está el ser humano; la persona, con sus capacidades intelectuales, su creatividad, su voluntad, su esfuerzo y tantas otras cualidades es capaz de descubrir, de inventar, de ordenar su entorno, en lo que consideramos, de forma positiva, un constante y mantenido progreso. Este progreso convive con episodios destructivos como las guerras, la violencia, el hambre o las enfermedades, pero sobresale y destaca lo positivo, porque son más los individuos dispuestos a ir hacia delante que los que destinan sus esfuerzos a obstaculizar este progreso.

De todo ello vamos a hablar en este artículo intentando identificar las características de la sociedad española del siglo XXI, del que ya llevamos casi tres lustros; esta radiografía de la sociedad actual, con sus necesidades y desafíos la pondremos frente a la Medicina del Trabajo, para destacar cómo ésta debe adaptarse y responder con todos sus recursos y posibilidades a una población trabajadora en constante cambio y con grandes expectativas respecto a su vida, su salud y su capacidad de disfrute.

\section{SOCIEDAD ESPAÑOLA ACTUAL Y CAMBIOS RECIENTES}

En la década de los años 90 encontramos ya una España sólidamente democrática, y con modelos sociales europeos muy incorporados y distintos a los vividos por la generación anterior. Este artículo no es un estudio sociológico, por tanto referiremos lo vivido por nosotros y documentado, en algunos de sus aspectos, en fuentes bibliográficas que han reconocido que el último cuarto del siglo XX en España ha estado plagado de constantes e interesantes cambios sociales, que continúan en la entrada en el siglo XXI ${ }^{1}$.

1 Así lo refleja la obra coordinada por Salustiano del Campo y José Félix Tenazos España siglo XXI, y cuyo primer volumen lleva por título La Sociedad, editada por Biblioteca Nueva, Madrid 2008. Los cuatro volúmenes restantes se dedican a la política, la economía, la ciencia y la tecnología y, por último, la literatura y las bellas artes. Esta obra resulta imprescindible para conocer bien la España de hoy y los cambios sucedidos desde la transición política de 1975. Para documentar cifras procedentes de estadísticas nacionales hemos acudido a la información que los respectivos Ministerios ponen a disposición del público en general (google.com). 
De estos cambios destacamos:

\section{II.1. Cambio demográfico}

A 1 de enero de 2013 la población española se estima en 47.129 .783 habitantes (Fuente: Instituto Nacional de Estadística), de los que 5.708 .940 (12,2\%) eran extranjeros. La distribución es muy irregular concentrándose en las costas, en los valles del Guadalquivir y del Ebro y en las llamadas áreas metropolitanas de las que sobrepasan los 700.000 habitantes las siguientes: Madrid (6.052.247), Barcelona (5.030.679), Valencia (1.551.585), Sevilla (1.294.867), Málaga (953.251), Bilbao (910.578), área central de Asturias (835.053) y Zaragoza (746.152).

Los avances de la medicina y la mejora de la calidad de la calidad de vida, en general, han aumentado las expectativas de vida que era de 73,7 años en 1975, para subir diez años más tarde a 76,4; en el año 2005 alcanzó los 80,3 años y en 2010 los 81,9. En el momento actual parece haberse estabilizado en 82 años, aunque con diferencias entre Comunidades Autónomas (Madrid: 83,7; Navarra: 83,4; Castilla y León: 83,2; Andalucía: 80,9 y Ceuta y Melilla con los valores más bajos están en 80,4). La diferencia por sexos es notable ya que para los varones la media está en 79,3 , mientras que para las mujeres llega a 85 .

Por otro lado, la natalidad ha experimentado una progresiva disminución en España; en 1995 alcanzó la caída máxima (363.467 nacimientos), para ir creciendo lentamente, alcanzando en 2008 los 518.503 nacimientos; sin embargo, de nuevo, en los años siguientes disminuyó el número hasta los 468.430 nacimientos en 2011, cayendo la media hasta 1,35 hijos por mujer, cuya edad media se elevó a 31,4 años. En 2013 nacieron en España 425.390 niños lo que significa una tasa de nacimientos de 9,13 por cada 1000 habitantes; esto significa un descenso del 6,43\% respecto al año 2012 y el $\mathrm{n}^{\circ}$ medio de hijos por mujer también descendió hasta el 1,7 (Fuente: Ministerio de Sanidad, Servicios Sociales e Igualdad). No podemos olvidar que la despenalización del aborto llevó a que en el año 1990 se produjeran en España 37.135 abortos, con cifras crecientes que han llegado en 2010 a 118.359, manteniéndose en los tres últimos años en cifras de alrededor de 120.000 (Fuente: Ministerio de Sanidad, Servicios Sociales e Igualdad).

Estos datos explican que de una población que se correspondía con una estructura, por edad, de forma piramidal hemos ido pasando a un gráfico en el que disminuye la base mientras crece el número de personas que alcanzan edades avanzadas, con un ensanchamiento central (forma de hucha), lo que se corresponderá con una sociedad progresivamente envejecida, según las previsiones, al menos hasta $2050^{4}$.

La situación descrita nos lleva a la preocupación sobre el futuro de las pensiones ante la disminución de la población activa que debe sostenerlas. Otros problemas a solucionar van paralelos a las necesidades de cuidados de las personas de edad avanzad, con mayor demanda de residencias y de cuidadores. Cuando el cuidado recae en los hijos (generalmente hijas), encontramos una situación impensable hace cincuenta años, y es que la cuidadora puede tener más de 65 o 70 años ya que la persona cuidada supera los 85 o 90 años.

\section{II.2. Cambio en los movimientos migratorios}

La prosperidad, el crecimiento de la economía española y la necesidad de trabajadores no cualificados en la agricultura, la construcción o el cuidado de personas mayores, sirvió de reclamo para trabajadores de otros países. En los años 80 y 90 fue constante la incorporación de mujeres de distintas nacionalidades (filipinas, ecuatorianas, colombianas, bolivianas, marroquíes o rumanas) para el servicio doméstico y el cuidado de las personas mayores. A partir de una inmigración unipersonal se fueron constituyendo estructuras familiares bien asentadas, ampliándose las de origen sudamericano a las marroquíes, y de otras poblaciones del este de Europa y del área subsahariana. Otro grupo racial importante ha sido la población china; se trata de una emigración familiar y vinculada al comercio 24 horas y sus proveedores, el cual ha ido desplazando al pequeño comercio español familiar y de barrio. 
En 2005 la tasa de inmigración en España era de 1,5\% anual, sólo superada por Chipre, actualmente está en un 0,99\%, ocupando el número 15 de la UE. En 2009 el 12\% de la población residente en España era de origen extranjero destacando los rumanos (796.576), marroquíes (710.401) y ecuatorianos (413.715); el 40,5\% del total de extranjeros procedían de la UE.

Si ponemos en relación la inmigración con la natalidad, encontramos que en el año 2000 nacieron en España 24.644 niños de madres inmigrantes, de ellos el 25.22\% eran marroquíes, el $10.53 \%$ eran ecuatorianos, el $6.15 \%$ eran colombianos, el $3.9 \%$ eran del Reino Unido, el 3.8\% eran chinos, el 3.4\% eran franceses, el 3.11\% eran portugueses (la mayoría corresponden a madres que vienen a parir a hospitales fronterizos por la mejor asistencia, volviendo después a su lugar de origen), el 3\% eran peruanos, y el 2.7\% era alemanes. (Fuente: Instituto Nacional de Estadística).

Se prevé que en el año 2.025 los inmigrantes serán el grupo laboral mayoritario; sin embargo, la crisis económica de 2008 ha provocado la vuelta a sus países de inmigrantes por falta de trabajo observándose en este momento un mayor ajuste entre la oferta y la demanda, circunstancia que puede volver a cambiar en años venideros.

\section{II.3. Cambio de los modelos familiares}

La introducción del divorcio en 1981, (con la reforma en 2005 con el llamado "divorcio exprés") produjo un aumento progresivo de rupturas familiares que llevaron después a nuevas reestructuraciones a través de segundos matrimonios o parejas; esto ha generado familias mixtas con convivencia en el hogar de menores sin vínculo de sangre entre ellos (hijos de anteriores matrimonios de los dos miembros de la nueva pareja). Otra novedad ha sido la aparición de familias monoparentales, sobre todo de mujeres solas, madres de hijos producto de técnicas de reproducción asistida o de adopción. También aumentó el reconocimiento social de las parejas homosexuales, constituidas bien por convivencia o incluso por matrimonio civil.

La adopción no ha podido producirse sobre niños de nacionalidad española por la baja natalidad ya comentada; la procedencia de los niños ha sido fundamentalmente de China, Hispanoamérica, Rusia, Países balcánicos y África; éstos niños se han integrado bien, pero se están incorporando ahora al estrato social adulto y está por ver las características de las familias que constituyan cuando corresponda, así como su desarrollo social futuro.

De la familia española de mitad del siglo XX en la que era habitual la convivencia en el mismo hogar de tres generaciones, estando los mayores al cuidado de los más jóvenes, se ha pasado a familias en las que los mayores viven solos, los hijos constituyen familias independientes, con baja natalidad, con un trato interno más igualitario, ya que la mujer, plenamente incorporada al mercado de trabajo, contribuye a la economía familiar y reclama un reparto de tareas y obligaciones domésticas más justo. En esta estructura familiar es frecuente que la generación intermedia, busque el apoyo de los padres como ayuda para el cuidado de los hijos menores ("abuelos esclavos"), habiéndose convertido en muchos casos, los abuelos y su pensión, en un sostén económico importante para los hijos en situaciones laborales precarias.

La separación y el divorcio de matrimonios jóvenes ha llevado a que, al menos en el periodo inmediato, con más frecuencia los hombres, vuelvan a vivir con los padres; esto se concreta en nuevas cargas, sobre todo para la mujer, que ya tiene cierta edad y había dejado atrás la etapa de la responsabilidad de cuidados sobre los hijos.

Todos estos cambios han sido bien aceptados por la sociedad española, y en las encuestas de opinión aparece reiteradamente la familia en el primer lugar de importancia para la mayoría de los encuestados, hecho que se ha afianzado aún más durante la reciente crisis. 


\section{II.4. Cambio cultural y educativo}

La emigración española desde zonas agrícolas, ocurrida en los años 50-60, hacia Alemania y Francia, no dio lugar a un cambio cultural para ellos o sus familias, ya que allí mantuvieron una vida aislada y austera. Simultáneamente se produjo otra emigración, de tipo nacional, más familiar, procedente sobre todo de Andalucía y Extremadura, hacia los territorios que gozaron del apoyo económico para la industrialización, como Cataluña, País Vasco y Valencia; aunque en los años 80 se produjo un declive industrial, la mayoría de las familias permanecieron allí, sobre todo en Cataluña donde la segunda generación ya se había estabilizado laboral y personalmente.

El cambio cultural vino de la mano del cambio político con ofertas en el ámbito artístico (teatral, musical, literario, pintura, arquitectura o museístico), o en la radio y TV, etc., haciéndose habituales, de forma progresiva, actitudes tolerantes en todos los ámbitos, hacia las formas de pensar y de actuar de los otros. Sin embargo, la sociedad española cambió más respecto a una apertura a las costumbres extranjeras, que en la incorporación generalizada de una auténtica inquietud hacia las artes y las letras, expresada en la lectura o la asistencia a actos culturales, que se mantuvieron en círculos elitistas y ligados a clases acomodadas.

Nuestra experiencia en violencia contra la mujer ${ }^{5}$ nos ha demostrado que los conflictos por maltrato en personas de más de 60 años tienen como origen el hecho de que las mujeres han sido más sensibles al cambio social de apertura y reconocimiento de derechos de la mujer, mientras que los hombres se han aferrado a la relación desigual, autoritaria y dominante tradicional, lo que ha acabado en muchos casos, en maltrato, denuncia y divorcio en edades avanzadas y después de muchos años de matrimonio.

El denominado postmodernismo llevaba consigo una actitud escéptica y en cierto modo irónica y hasta frívola hacia el entorno real. Para muchos se ha tratado de un movimiento vacío de ideas y gran pobreza creativa, sin que se hayan producido en la etapa final del siglo XX y principios del XXI grandes figuras de la filosofía, la literatura, la pintura o la música. En el año 2000 se empieza a hablar del "posmilenarismo", considerando que hay que superar una actitud victimista, olvidando el resentimiento y las rememoraciones de los agravios pasados para ir a un diálogo de encuentro auténtico ${ }^{6}$.

En el momento actual se acepta que la denominada "crisis económica" lleva aparejada una crisis más extensa que afecta a los aspectos ético-morales de las personas con una evidente crisis de valores y virtudes que deben recuperarse ${ }^{2}$. Por ello, la sociedad española (como la europea) está a la búsqueda de nuevas ideas que orienten las inquietudes socioculturales, proponiéndose objetivos "universales" sobre los que trabajar como el cambio climático, la conservación del planeta o las altísimas desigualdades entre las poblaciones (hambre, infecciones, violencia, etc.) lacras que habría que combatir y erradicar.

En general los españoles hemos viajado más y hemos conocido mejor Europa y el mundo pero no disfrutamos, en el momento actual, de movimientos culturales bien identificados y consistentes en su dirección y contenidos.

En el aspecto político destaca la aparición de una importante tendencia antisistema, sobre todo entre los más jóvenes que se está organizando políticamente con planteamientos muy radicales. Sociológicamente se interpreta como una reacción debida a la decepción ante comportamientos políticos corruptos y de incumplimiento de promesas electorales. También alertan los sociólogos del aumento de posturas antirracistas y xenófobas ante la creciente inmigración ilegal. Esto contrasta con el extraordinario espíritu de solidaridad que la sociedad española manifiesta ante catástrofes puntuales, y de forma continuada con altísimo compromiso de voluntariado y pertenencia a ONGs; el número de misioneros españoles desplazados a territorios con alto grado de pobreza o enfermedad está en

${ }^{2}$ Como ejemplo de olvido ético cotidiano pueden servir los programas televisivos denominados realityshow, en los que se maltrata la intimidad o la dignidad personal al colocar a personas que, a menudo están en situación límite en su economía o su salud mental, ofreciéndoles la oportunidad de desnudarse íntima y moralmente ante millones de espectadores, con la finalidad económica en primer plano. 
los 13.000, siendo aún más amplio aunque más cambiante el número de voluntarios ${ }^{3}$. Podemos hablar por tanto de la existencia de una cultura de ayuda solidaria y caritativa en la sociedad española hacia los que viven entre nosotros y hacia otros países.

En el aspecto educativo, el gran logro había sido el destierro del analfabetismo. La escolarización y enseñanza obligatoria igualó el nivel educativo de los españoles en nuestra etapa democrática. Políticamente España ha adolecido de un pacto de Estado en educación, lo que ha originado que la alternancia de gobiernos haya generado siete leyes educativas (dos con UCD, cuatro con el PSOE y la más reciente del PP). La LODE (1985) introdujo el modelo de colegios concertados; la LOGSE (1990) introdujo la escolaridad obligatoria hasta los 16 años y dio gran peso educativo a las CCAA; la LOPEG (1995) fue rechazada por los sindicatos entendiendo que potenciaba la privatización; La LOE (2006) introdujo la asignatura Educación para la ciudadanía, muy contestada por algunos de sus contenidos y la diversa orientación que recibía en cada CA, y porque iba en detrimento de Lengua y Literatura, disminuyendo la exigencia al aceptar el paso de curso con asignaturas suspensas; la LOMCE (2013) se ha comenzado a aplicar el presente curso con mucha oposición, recupera mayor exigencia con pruebas externas de evaluación o reválida. (Fuente: Ministerio de Educación y Ciencia.)

Los cada vez menos exigentes estudios han dado lugar a que los escolares españoles aparezcan mal calificados en estudios comparativos con estudiantes europeos próximos a finalizar la enseñanza obligatoria en áreas temáticas como las ciencias, las matemáticas o la lectura ${ }^{4}$.

En el nivel universitario, España ha crecido en cantidad, pero no en calidad. Existen actualmente universidades en todas las provincias españolas, y en estudios como los de Medicina contamos con 40 facultades; las últimas 12 se han abierto en los últimos cinco años, siendo 9 de ellas privadas; estas cifras nos colocan como tercer país del mundo en número de facultades de Medicina, detrás de Corea y Sudán; de hecho se forman alrededor de 2.500 médicos más de los necesarios, lo que genera un excedente que acaba buscando fuera de nuestras fronteras su futuro profesional. (Fuente: Ministerio de Educación y Ciencia.)

En el momento actual la enseñanza universitaria presencial, aprovechando la tecnología informática, se complementa con la docencia on line, a través de la cual se imparten titulaciones de grado, de master y de especialización llevando los conocimientos al propio domicilio de la persona que se forma, promoviendo su participación en el propio proceso formativo.

Nuestra ciencia ha sido parca en premios Nobel (Santiago Ramón y Cajal de Medicina y Severo Ochoa de Fisiología y Medicina), sin embargo, nuestros médicos, enfermeras, fisioterapeutas, informáticos o ingenieros son bien recibidos en Europa y el mundo y allí, una vez que nos hemos gastado nuestros recursos en su formación, desarrollan sus capacidades al máximo y dejan sus frutos, por la falta de trabajo para ellos entre nosotros ${ }^{5}$.

Los idiomas siguen siendo un punto negro en el ámbito educativo español; en los casos en los que mejora es a costa del pecunio familiar y como estudios complementarios;

3 Organizaciones como Cáritas, Cruz Roja, manos unidad, médicos mundi o enfermeros mundi pone de manifiesto esta realidad. La Organización Médica Colegial (OMC) viene entregando en los últimos años un premio a la "cooperación" dentro de los premios a la "trayectoria profesional"; este premio resulta altamente reñido por el alto número de profesionales médicos que se desplazan a países en desarrollo (generalmente los más pobres) con proyectos de tipo quirúrgico, asistencial en el ámbito de las enfermedades infecciosas, o de asistencia a las mujeres y la infancia, para ello es frecuente que empleen sus periodos de vacaciones o tiempo no remunerado.

${ }^{4}$ Así lo demuestra el Programme for Internacional Student Asseessment, conocido entre nosotros como Informe PISA.

5 Esta política necesita revisión urgente por la incongruencia social y económica que supone; si no hay puestos de trabajo para estos profesionales no podemos crear costosos centros formativos para ellos; el presupuesto dedicado a su formación se debe invertir en cambiar la estructura económica para que genere puestos de trabajo, quizá a niveles de titulaciones medias, en lugar de generar titulados superiores para exportar. 
en nuestros colegios e institutos no se estudia en dos o más idiomas como sucede en el resto de Europa y, en los lugares en los que se enfatiza el estudio de otra lengua suele ser la autóctona (catalán, vasco, gallego o valenciano) y en detrimento del español como primera lengua nacional, lo que, en lugar de abrir puertas al mundo, las cierra ${ }^{6}$.

\section{II.5. Cambio en la situación económica}

En el momento actual España ocupa el lugar décimosegundo de la economía mundial. El último cuarto de siglo se había caracterizado por un crecimiento mantenido con expresión en una construcción exuberante que alcanzó precios muy elevados, a pesar de lo cual una gran mayoría de familias españolas accedieron a la segunda vivienda apoyándose en créditos bancarios.

La crisis de 2008 ha sido devastadora en todos los ámbitos, pero ha sido la construcción expansiva y el estallido de la "burbuja inmobiliaria" el símbolo más evidente de la mencionada crisis. La pérdida de empleo fue progresiva con el consiguiente impago de las hipotecas y el endurecimiento de la concesión de créditos, lo que bajó drásticamente la venta de viviendas con la caída de la construcción y empresas auxiliares; lo siguiente fue la suspensión de pagos de las constructoras, lo que supuso para la banca recuperar lo prestado en forma de viviendas ("activos tóxicos"), siendo necesaria una reestructuración bancaria (Cajas de Ahorros) que todos hemos contribuido a paliar. El rescate bancario ha tenido como objetivo colocar a la banca en disposición de volver a conceder créditos, sobre todo para los autónomos y pequeña y mediana empresa, que son las estructuras que más empleo generan en nuestro país, así como a las familias; se espera que cuando esto suceda se dinamizará el emprendimiento y la economía.

En el ámbito de la economía, la agricultura ofrece, actualmente, productos estrella como las verduras y hortalizas de invernadero, el aceite de oliva, los vinos y los cítricos, aunque esta sólo significa el 5\% del PIB; la comercialización alimentaria está en su mayor parte en manos de industrias francesas. La ganadería ha pasado, en gran parte, al modelo estabulario. En 2005 la cabaña ganadera alcanzaba los 22,7 millones de ovino, 25,1 millones de porcino, 6,5 millones de vacuno y 3 millones de caprino, junto a 131 millones de aves de corral. Sin embargo, el ingreso en la UE, significó para la ganadería española la disminución, sobre todo de vacuno para asumir la producción de leche de otros países como Francia. También la pesca ha disminuido en los últimos años a pesar de la importancia de nuestra flota y de la excelente preparación de sus pescadores; las capturas totales suponen el $60 \%$ de la UE, casi 230.000 toneladas, aunque siempre estamos pendientes de los acuerdos con Marruecos y otros caladeros más lejanos, que gestiona la UE. (Fuente: Ministerio de Agricultura y Pesca). La minería ha sido otro sector económico venido a menos. El carbón español tiene menos rentabilidad que el europeo por lo que su extracción prácticamente ha desaparecido; sigue siendo rentable la sal y los minerales de hierro, cinc, plomo y rocas industriales.

Al constituirse la Comunidad Económica Europea, España tuvo la consideración de "país de servicios" debido a su geografía marítima y buen clima. Esto ha favorecido que el turismo haya sido el motor de la economía española en los últimos años, en constante crecimiento y con una cuota del 6\% del turismo mundial; en 2012 fuimos el cuarto país en la recepción de turistas para ser en 2013 el segundo, junto a EEUU, después de Francia; esto supuso cifras superiores a los 56.000 millones de euros. Las Comunidades Autónomas más visitadas han sido Cataluña, las Islas Baleares y Canarias, Andalucía, la Comunidad Valenciana y Madrid; en 2013 se han superado los 60 millones de personas (Fuente: Ministerio de Industria, Energía y Turismo.)

${ }^{6}$ Es adecuado e importante mantener y cultivar las lenguas autóctonas, tradicionales y familiares, pero no a costa de renunciar a hablar bien otra lengua mucho más vinculante con otros pueblos y países; el español lo hablan millones de personas en el mundo, y el inglés millones, habiéndose equiparado al latín vulgar medieval como vehículo verbal de entendimiento, consideramos, por tanto que estas dos lenguas deberían ser habladas por los escolares españoles al llegar a la universidad, además de la propia del territorio, en el caso de que la tengan. 
Económicamente, la situación de crisis de los últimos años ha generado un altísimo nivel de desempleo que llegó al 26\%; esto ha obligado a ajustes presupuestarios con bajadas de sueldos sobre todo en la administración, congelación de salarios y subidas de impuestos, lo que ha generado un deterioro económico familiar. Los ajustes presupuestarios han significado recortes importantes en todos los ámbitos siendo más contestados en la sanidad o la educación.

La pertenencia a la UE y su moneda única nos ha dado estabilidad; al parecer, la recuperación se va produciendo con datos macroeconómicos progresivamente mejores, aunque el reto sigue siendo la creación de empleo.

\subsection{Cambio tecnológico}

Aunque pueda parecer que España no es un país que genere alta tecnología, es una impresión equivocada. Somos líderes en el ámbito de comunicaciones y telefonía, así como en energías renovables y construcción de parques eólicos, energía termosolar e industrias desalinizadoras. La red ferroviaria de alta velocidad de la que disfrutamos ha sido reclamo para que empresas españolas vayan a construir líneas como Ankara-Estambul o La Meca-Medina. España es el primer país en Agrobiotecnología y el $5^{\text {a }}$ de Europa en bioquímica y biología molecular y producción de hemoderivados; también en Europa ocupa el $1^{\circ}$ lugar como fabricante de vehículos industriales y el $2^{\circ}$ lugar en automóviles y está entre los tres primeros en fabricación de buques. Respecto al ámbito de la ingeniería informática ocupa un importante lugar en el desarrollo de sofware antivirus o control de satélites. Actualmente tenemos dos bancos entre los 20 primeros del mundo (BS y BBV).

El descubrimiento del ADN y los estudios de genética individual y poblacional nos han permitido conocer mejor a los seres vivos y, particularmente a los seres humanos y, sobre todo, el origen de numerosas enfermedades; España ha incorporado estas tecnologías y tiene numerosos centros de referencia para laboratorios clínicos y forenses, sobre todo de Hispanoamérica.

En general, aprovechamos todos los avances producidos en el ámbito de la ingeniería informática, las comunicaciones electrónicas y las redes sociales para promoción de ideas personales que los nuevos emprendedores van concretando en negocios diversos que se abren camino en los diferentes ámbitos del mercado, como ejemplo tenemos las numerosas páginas web a través de las que promocionan y venden sus productos, desde pequeños ganaderos, agricultores, empresas tecnológicas y de servicios, o profesionales entre otros.

Como resumen, España progresivamente se incorpora a los procesos de $\mathrm{I}+\mathrm{D}+\mathrm{I}$ (Investigación/Desarrollo/Innovación) con previsible crecimiento en un futuro próximo.

\section{POBLACIÓN TRABAJADORA EN ESPAÑA: POBLACIÓN ACTIVA Y POBLA- CIÓN DESEMPLEADA. POBLACIÓN JUBILADA}

Según la Encuesta de Población Activa (EPA) en el segundo trimestre de 2014 el número de personas ocupadas en España era de 17.353 mientras que el de personas en paro era de 5.622,9 millones.

La propuesta para la clasificación de las clases sociales conocida como "Clasificación Socioeconómica europea (ESeC) distingue: a) Grandes empleadores, directivos y profesionales de nivel alto, b) Directivos y profesionales de nivel bajo, c) Empleados de cuello blanco de nivel alto, d) Pequeños empleadores y trabajadores autónomos no agrícolas, e) Trabajadores autónomos agrícolas, f) Supervisores y técnicos de rango inferior, g) Trabajadores de los servicios y comercio de rango inferior, h) Trabajadores manuales cualificados, i) Trabajadores no cualificado y j) Excluidos del mercado de trabajo y parados de larga duración (7). Los datos encontrados sobre el mercado de trabajo en España no se corresponden exactamente con esta clasificación, ya que aparecen agrupados en los sectores "agrario" y "no agrario", y dentro de éste se diferencian 
"industria", "construcción" y "servicios", quedando fuera los trabajadores de la administración pública. (Fuente: Ministerio de Empleo y Seguridad Social.)

\section{Población activa}

De 2011 a 2012 se produjo una disminución de población empleada, que se concreta en un 3,5\% en mujeres y un 6,5\% en hombres; disminuyó más el empleo temporal $(12,4 \%)$ que el indefinido $(2,9 \%)$ y el tiempo completo $(6,6 \%)$ que el trabajo a tiempo parcial $(0,4 \%)$.

Respecto al tipo de trabajo, fue la construcción donde el empleo se redujo más, con un $20,5 \%$; en la industria fue de un $6,4 \%$ y en el sector servicios un 3,4\%. Destaca en la pérdida de puestos de trabajo el tamaño de la empresa siendo del $4,3 \%$ en las de menos de 10 trabajadores; fue del 7,9\% en empresas entre 11 a 50 trabajadores, del 4,6\% en las de más de 250 trabajadores y del 3,5\% en las de 51 a 250 trabajadores.

Así a final de diciembre de 2012, (excluyendo el sector agrícola y la administración pública) estaban empleados 10,92 millones de personas, de los que 5,702 eran varones y 5,215 eran mujeres; siendo el 78,4\% contratos indefinidos y un 21,6\% temporales. De ellos el $77,2 \%$ el trabajo era a tiempo completo y el resto a tiempo parcial. (Fuente: Ministerio de Empleo y Seguridad Social.)

\section{Población desempleada}

El paro registrado en diciembre de 2013 ofrece las siguientes cifras: 4.701,3 millones de personas de las que los varones eran 2.294,7 y las mujeres 2.4066,6. En agosto de 2014, el número de desempleados era de 4.427,9, siendo varones 2.099,8 y mujeres 2.328,1. La tasa de paro en el segundo trimestre de 2014 ha sido del 24,47.

De los desempleados jóvenes encontramos en diciembre de 2013, en el grupo de menores de 25 años 412,6 y entre 25 y 44 años la cifra era de 2.311,0 personas. En agosto de 2014, los menores de 25 años desempleados eran 386,3 y la cifra de los que tenían entre 25 y 44 años era de 2.095,1.

De los desempleados actualmente, unos dos millones tienen baja cualificación correspondiéndose con los que abandonaron sus estudios para trabajar en la construcción, sector en el que más cayó el empleo.

En términos generales se puede resumir que 1/4 parte de la población activa está desempleada, y de ella $1 / 2$ son jóvenes.

\section{Población jubilada}

La edad media de jubilación en España ha subido, por primera vez a 64 años, en 2013. En general, varía entre los 70 años (en profesiones intelectuales como la universidad, judicatura), pudiendo incluso prolongarlo con nombramientos de eméritos, frente a las prejubilaciones de otros sectores con actividades de mayor desgaste físico, aunque las jubilaciones más juveniles se han producido como consecuencia de la reestructuración de la banca, a una edad media de 52 años; los 65 años se establecen para los docentes de la enseñanza media y la función pública, aunque aumentado en unos meses tras la reforma del anterior gobierno.

Los jubilados forman un grupo numéricamente importante que para muchos ha comenzado con poco más de 50 años, circunstancia que les ha permitido encontrarse con alta disposición de tiempo para actividades no laborales (universidad de mayores, UNED, viajes culturales, voluntariado, pertenencia a ONG, etc.), aunque se encuentran excluidos de diversos ambientes sociales.

En los presupuestos generales del Estado de cada año, asistimos al debate del reparto equilibrado entre ingresos y gastos, con grandes capítulos de gasto en pensiones, 
desempleo, sanidad, educación o servicios sociales, mientras que los ingresos los integran las cotizaciones a la Seguridad Social de las personas empleadas y los impuestos.

Las pensiones mencionadas y, en especial las de jubilación (contributivas y no contributivas), significan la gran preocupación de un gobierno que desee mantener el Estado del Bienestar. En 1995 el llamado Pacto de Toledo estableció un acuerdo político que compromete a los gobiernos a mantener una caja (o hucha) de garantía del pago de las pensiones para los años venideros. Sin embargo, de forma constante se nos recuerda la preocupación de que es necesario un número suficiente de trabajadores cotizantes a la SS para que las pensiones puedan garantizarse con todos los derechos ciudadanos que llevan aparejados lo que puede verse amenazado si se descontrola el desempleo.

En la población descrita para los lustros venideros observamos una población envejecida (8) de la que se nos indica que si en el año 2007 cada persona empleada sostenía a 1,2 personas en situación de dependencia, y en el año 2010 esta cifra se elevaba a 1,5, en el 2015 estaremos ya en 1,7. La necesidad en pensiones se estima en 130.000 millones de euros, en los presupuestos generales para el 2015. El 4 de octubre de 2014 el Ayuntamiento de Madrid en el "día de las personas mayores" rindió homenaje a 1.217 personas con más de 100 años, siendo dos mujeres de 106 años las de mayor edad.

La baja natalidad en España hace prever que en el año 2050 la población con más de 65 años llegará al 68\%.

La realidad es que estos datos ponen de manifiesto un perfil de población activa y un mercado laboral absolutamente novedoso, que merece una profunda reflexión para, de un lado mitigar el número de desempleados, y de otro, mantener el número de trabajadores, y aumentar el periodo de vida laboral para controlar el número de pensionistas.

\section{SINIESTRABILIDAD LABORAL}

La patología laboral por excelencia se concreta en los Accidentes de Trabajo (AT) y las Enfermedades Profesionales (EP).

\section{IV.1. Siniestralidad y Accidente de Trabajo}

El AT definido como "toda lesión que el trabajador sufre con ocasión o por consecuencia del trabajo...", ocurre de forma imprevista y puede deberse a los más diversos mecanismos, externos o venidos de fuera, con resultados que pueden ir desde un grado leve que no requiere baja laboral, hasta producir la muerte. En España el accidente in itínere tiene legalmente la condición de AT, ya que se produce al ir o volver del trabajo; sin embargo, de cara a la acción preventiva que puede hacerse para evitar los AT desde la Medicina del Trabajo, la Seguridad y la Higiene en el trabajo, la posibilidad de actuación sobre estos accidentes de tráfico, en los que influyen circunstancias ajenas al ambiente de trabajo, es muy escasa; por este motivo, algunos países consideran al accidente in itínere como una situación que puede ser indemnizada de manera particular, pero sus cifras no "engrosan" las de los accidentes ocurridos en el lugar y en el tiempo del trabajo sobres los que debe trabajarse intensamente con perspectiva preventiva.

De todos los datos de AT que el Ministerio de Empleo y Seguridad Social pone a nuestra disposición destacamos los siguientes:

Respecto a los AT "mortales" en el periodo comprendido entre enero-julio de 2013 se produjeron 260, cifra que ha pasado a ser de 273 en el mismo periodo de 2014, (13 más), éstos se distribuyeron en 46 en el sector agrario, 59 en la industria, 35 en la construcción y 133 en el sector servicios; en su conjunto, significan un aumento del 3,6\%.

En los mismos periodos de enero-julio de 2013 y de 2014 tenemos que el número de AT con baja fue en 2013 de 266.764, pasando a 279.183 en 2014 (37.089 in itínere). De los 242.094 ocurridos en tiempo de trabajo, 1870 fueron "graves", cifra que disminuyó en 
19 respecto a los ocurridos en el mismo periodo de 2013 con la misma calificación (1889). Con la calificación de "leves", en 2013, ocurrieron 228.610, aumentando a 239.951 en 2014 (11.341 más); de manera que considerando todos los AT con baja de 2014 se produjo un aumento del $4,7 \%$, respecto al mismo periodo del año anterior.

En la distribución por sexo, de los ocurridos en 2014 en tiempo de trabajo, 166.173 $(68,6 \%)$ correspondieron a varones, mientras que $75.921(31,4 \%)$ correspondieron a mujeres.

La incidencia entre enero-julio de 2014 respecto al mismo periodo del año anterior ofrece una media mensual de 254,8 accidentes por cien mil trabajadores, lo que significa un aumento del 3,6\%.

Este mismo dato referido por sectores encontramos que la del sector agrario fue de 392,4 accidentes por cien mil trabajadores en 2014 lo que significó un aumento del 4,4\%; en la industria fue de 396,6, lo que significó un aumento del $2 \%$; en el sector servicios fue de 205,4 lo que significó un aumento del 4,1\% y en la construcción fue de 504,2, significando un aumento del 6,3\% respecto al mismo periodo del año anterior.

Los AT sin baja entre enero-julio de 2013 fueron de 394.486 pasando en 2014 a 398.069.

Los sindicatos atribuyen estos aumentos a la precariedad laboral generada por la crisis, habría que conocer los datos de cada accidente para tener la certeza de las causas que están detrás de cada siniestro laboral, en los que, sin duda influye la formación y capacidad del trabajador, junto a factores de seguridad, bienestar y tranquilidad en el desempeño de su tarea.

\section{IV.2. Siniestralidad y Enfermedad Profesional}

El actual cuadro de EP es el contenido en el RD 1299/2006, de 10 de noviembre, que establece, también, los criterios para su notificación y registro. Este se complementa con la Orden TAS/1/2007, de 2 de enero; en ella se encomienda a los médicos de Atención Primaria la función de sospecha y detección del origen laboral de la patología por la cual el paciente consulta, estableciendo la forma en que estos médicos deben cursar electrónicamente el Parte de Enfermedad Profesional para su posterior gestión por la entidad que corresponda (artículo 5 del RD 1299/2006).

Cada Comunidad Autónoma ha dispuesto el organismo que actúa como intermediario entre el médico del Sistema Nacional de Salud (Médico de Familia o especialista) y el INSS (como entidad gestora), o la MATEPSS (como entidad colaboradora) para que, confirmado el diagnóstico y calificada la patología como EP, se proceda a su registro con las consecuencias previstas de tipo administrativo y preventivo.

La cumplimentación y transmisión del parte de enfermedad profesional se realizará, únicamente por vía electrónica, por medio de la aplicación informática CEPROSS (Comunicación de enfermedades profesionales, Seguridad Social).

De esta normativa se esperaba un aumento de diagnóstico de las EP, ya que existía la unánime opinión sobre su infradiagnóstico.

Los datos obtenidos del Ministerio de Empleo y Seguridad Social nos indican que las EP diagnosticadas fueron: 17.061, en 2007; 18.700 en 2008; 16.850 en 2009; 16.928 en 2010; 18.121 en 2011; 15.711 en 2012 y 16.901 en 2013.

Según la información de CEPROSS sobre la distribución de los Partes de EP comunicados entre enero y agosto de 2014, por grupos de enfermedad fueron: El 82,64\% correspondieron al Grupo 2 (enfermedades causadas por agentes físicos); el 5,21\% correspondieron al Grupo 5 (enfermedades de la piel causadas por agentes no comprendidos en alguno de los otros apartados); el 5,14\% correspondieron al Grupo 3 (enfermedades causadas por agentes biológicos); el 4,05\% correspondieron al Grupo 4 (enfermedades causadas por inhalación de sustancias y agentes no comprendidos en 
otros apartados); el 2;62\% correspondieron al Grupo 1 (enfermedades causadas por agentes químicos) y el $0,34 \%$ correspondieron al Grupo 6 (enfermedades causadas por agentes carcinógenos). De la diferencia entre sexos sólo llama la atención que el porcentaje en el Grupo 3 fue del 3,68\% en los hombres y del 6,69\% en los hombres, circunstancias que es coherente con el hecho de que los riesgos biológicos están en el ámbito asistencial sanitario, en el que predominan las mujeres, sobre todo enfermeras y auxiliares de enfermería, profesión que asume un riesgo biológico mayor.

En cualquier aspecto de la siniestralidad laboral la prevención es posible y obligada, pero aún lo es más en el ámbito de las EP ya que se trata de actividades cuyo riesgo es conocido y debe ser evaluado y controlado; en derecho se dice que "lo que es previsible debe ser evitado" y este precepto aquí cobra especial relevancia.

\section{LA MEDICINA DEL TRABAJO EN EL SIGLO XXI}

La Real Academia de Medicina de Andalucía Oriental inauguraba en Granada el 2 de octubre de 2014 la exposición titulada "100 años de Medicina en España", cuyo contenido se publicaba en la Revista Actualidad Médica9. Por especialidades y sintéticamente se destacan los hitos que han apoyado el extraordinario progreso médico en este periodo; la característica más común es la rapidez con que todo sucede reduciéndose el tiempo de los cambios a días. Por su importancia destacamos el éxito de los trasplantes de órganos que mantienen a España en un primer lugar a nivel mundial, el control del VIH convertido en una enfermedad crónica, los avances en el conocimiento y curación del cáncer o de la hepatitis a través de terapias farmacológicas y físicas innovadoras, o la cirugía, que a través de las técnicas laparoscópicas y robóticas nos ha llevado al concepto de "cirugía ambulatoria". El mejor conocimiento genético con proyectos universales como el proyecto genoma humano, o el actual proyecto macrobiota humana ${ }^{7}$, que lleva a concebir la persona con su propio genoma y los genomas de sus microorganismos, promete conocer mejor numerosas enfermedades, lo que, sin duda ofrecerá oportunidades diagnósticas y terapéuticas.

La Medicina del Trabajo, para el cumplimiento de sus objetivos de preservar la salud de los trabajadores mediante la prevención y atenderlos cuando sufrieran lesiones o enfermedades, ha incorporado todos los avances producidos en el resto de las especialidades médicas basando en ellos su propio progreso, aunque en esta especialidad también se han producido sus propios cambios apoyados en la normativa legal y la mejora de la especialización.

\section{V.1. Normativa legal}

España incorporó la Directiva 89/391 de la CEE y del Convenio 155 de la OIT sobre seguridad y salud de los trabajadores y medio ambiente con la Ley 31/1995 de Prevención de Riesgos Laborales y el posterior Reglamento de los Servicios de Prevención. Con ellas se introducía la "vigilancia de la salud" como mecanismo preventivo de Enfermedades Profesionales (EP) y de Accidentes de Trabajo (AT). Junto a los Médicos del Trabajo se incorporan otros profesionales sanitarios como los Enfermeros del Trabajo, los Técnicos en Prevención, los Higienistas o los Psicólogos laborales. De estos equipos multiprofesionales se espera que generen en las empresas conductas orientadas a la preservación de la salud general y de la salud laboral, en particular, así como actitudes de seguridad en todas sus actividades.

El llamado Informe Durán realizado en 2001, por encargo del Gobierno ${ }^{10}$, puso de manifiesto que la nueva normativa no había conducido, aún, a la disminución de la

7 El día 7 de octubre de 2014, el profesor Manuel Díaz Rubio, dictaba en la Real Academia Nacional de Medicina una Conferencia sobre la enfermedad de intestino irritable en la que puso de manifiesto la importancia que tendrá en el futuro los resultados del proyecto internacional sobre el estudio genómico de la macrobiota humana. 
siniestralidad laboral, por lo que incluyó entre sus propuestas de mejora la referida al campo de la formación en seguridad y educación para la salud, la cual proponía: Formación adecuada de los Especialistas en Medicina del Trabajo, de los Técnicos en Prevención, de los Técnicos en Seguridad e Higiene Industrial. Habría que incluir también la sensibilización de los médicos de Atención Primaria respecto a la patología profesional. También se incidía en la formación y educación de los trabajadores en seguridad y salud laboral transmitiéndoles una cultura de prevención a través de la información sobre los riesgos/beneficios de las acciones preventivas.

El 25 de julio de 2014 publicaba el BOE el Proyecto de Ley sobre la modificación del régimen jurídico de las Mutuas de Accidentes de Trabajo y Enfermedades Profesionales, iniciando su trámite parlamentario “... con el objetivo de modernizar el funcionamiento y gestión de estas entidades privadas, reforzando los niveles de transparencia y eficacia, y contribuyendo en mayor medida a la lucha contra el absentismo laboral injustificado y a la sostenibilidad del sistema de la Seguridad Social...". Se pretende que ayuden al control de la Incapacidad Temporal (IT) ahora responsabilidad, casi exclusiva de los Médicos de Atención Primaria, aunque también se espera de esta norma que genere para los trabajadores mejor protección de la que ya tienen.

\section{V.2. Mejora de la especialización}

La especialidad de Medicina del Trabajo mantenida como especialidad de Escuela Profesional pasó en 2005 al modelo hospitalario común al resto de especialidades, proceso que contó con la colaboración económica de las Mutuas de Accidentes de Trabajo y Enfermedades Profesionales (MATEPS), los Servicios de Prevención de Riesgos Laborales (SPRL), Sociedades de Prevención y las administraciones a través de sus Institutos de Prevención. Así, los Médicos del Trabajo se integraron en la asistencia hospitalaria y en la específica de los Servicios de Salud Laboral hospitalarios y, en general, en la red asistencial promotora de la salud laboral en todos los ámbitos ${ }^{11}$.

La Convocatoria MIR de 2015 será la primera en la que se acceda a las especialidades médicas a través de dos años comunes en los troncos Médico, Quirúrgico, Laboratorio e Imagen, según el Real Decreto 639/2014 de 28 de julio, de nominado de Troncalidad, la Medicina del Trabajo está en el "tronco médico". Una Comisión ad hoc elaborará el programa definitivo de formación troncal, sobre un borrador previo ya existente. En breve se nombrarán las nuevas Comisiones de las Especialidades que deberán elaborar los programas específicos de cada especialidad para los años complementarios a los dos de troncalidad. Como todos los cambios la implantación de este nuevo sistema normativo, tendrá sus dificultades, pero confiamos en que el cambio traerá mayor igualdad aún, entre la Medicina del Trabajo y el resto de las especialidades médicas.

Todo lo anterior garantiza que en el futuro seguiremos contando, más aún si cabe, con Médicos del Trabajo competentes para mantener la salud de los trabajadores en el mejor estado posible, así mismo se mantendrá la tarea preventiva con los exámenes médicos indicados a cada situación, y la asistencia que corresponda en los casos de accidentes de trabajo y enfermedades profesionales, a nivel especializado.

\section{SITUACIÓN ACTUAL. PROPUESTAS PARA UN AFRONTAMIENTO IMAGINATIVO}

Las pinceladas expuestas sobre la sociedad española del siglo XXI con especial mención a los aspectos laborales, puede resumirse en:

1. Contamos con una población envejecida con un número creciente de personas con derechos sociales a pensión, a asistencia sanitaria, a actividades culturales y de ocio y a los cuidados y atenciones que necesiten según su estado de dependencia. Sin embargo, la situación descrita obligará en un tiempo breve a aumentar la edad de jubilación, probablemente hasta los 67 años, manteniendo la de los 70 para aquellas profesiones en 
las que actualmente esto es así. El tener en el futuro trabajadores mayores aconsejaría, desde la salud laboral el ir hacia un sistema de vida laboral más libre y personalizado, lo que podríamos denominar "la jubilación a la carta" o adaptada a las posibilidades y circunstancias de cada trabajador. Esto significaría que, en la medida en que la persona conservara su capacidad pudiera permanecer en el puesto de trabajo, aunque adaptada a un calendario de dedicación a tiempo parcial. Esta propuesta permitiría que se fueran integrando en el mercado laboral trabajadores jóvenes que, admitiendo incluso los contratos a tiempo parcial, realizarían un aprendizaje y una adaptación progresiva a sus tareas, aunque se debería procurar que esto sucediera en el menor tiempo posible y con la estabilización como meta.

\section{La población potencialmente activa se caracteriza por:}

Un número muy importante de titulados universitarios, en muchos casos excedentes, en relación con las necesidades laborales del momento (médicos, enfermeras, ingenieros, biólogos o químicos, entre otros), aunque esto sucede porque el ajuste económico dispone que la "tasa de reposición" de las jubilaciones sea del 10\%, por ejemplo en la docencia universitaria y otros sectores de la administración. Entre los médicos, por ejemplo, se producirán jubilaciones masivas en unos años y quizá tengamos que reclamar la vuelta de los que ahora buscan su futuro fuera de nuestras fronteras.

Otro grupo importante se corresponde con trabajadores no cualificados; su procedencia está en los jóvenes que dejaron los estudios para trabajar en la construcción, en los años 90, habiendo quedado desempleados tras la caída de este sector; también pertenecen a este grupo de baja cualificación la mayor parte de los trabajadores extranjeros, preparados para la agricultura, la construcción o los oficios auxiliares. No obstante, aunque serán los trabajadores cualificados los que antes ingresen en el mercado laboral, también les llegará el momento a medio plazo a los no cualificados. Hay indicadores de que muchos de estos jóvenes no cualificados han vuelto a los estudios o formación, lo que sin duda aumentará sus oportunidades.

El grupo de trabajadores de la banca está muy renovado y manifiesta predominio femenino, su juventud se debe a que las jubilaciones se han producido a edades más tempranas (prejubilaciones).

Las administraciones públicas también están reestructurando sus plantillas, pero aún emplean a un número muy importante de trabajadores cuyas jubilaciones se producen en los 65 años.

El grupo de los profesionales es muy diverso, por ello la jubilación también debería serlo. Ya hemos dicho que los profesores universitarios y los profesionales de la judicatura se jubilan a los 70 años, mientras que los profesores de enseñanza media lo hacen a los 65, al igual que los médicos de la asistencia pública. En el ejercicio libre la jubilación es más libre y se asimila a la de trabajadores autónomos. Las predicciones de envejecimiento ya comentadas plantearán a corto plazo la necesidad de afrontar un mayor número de pensiones y a cobrar durante más tiempo. Esto significa que, año a año, se necesitará una partida presupuestaria mucho mayor que la actual para el mantenimiento de los derechos sociales.

Los gobiernos tienen la obligación de aportar las soluciones necesarias. Es unánime la opinión de que será necesario alargar el periodo de vida laboral, circunstancia que ya se puso en marcha en la anterior legislatura (PSOE) y que habrá que aumentar, con toda probabilidad hasta los 67 o más años. El hecho de que sean necesarios más cotizantes a la SS indica que hay que aumentar la población trabajadora, hecho que debido a nuestra baja natalidad nos llevará a recurrir al aumento de la inmigración, que se hará necesaria una vez que las jubilaciones de los próximos años vayan absorbiendo a la población joven desempleada actualmente. 


\section{Propuestas de futuro}

Para la Medicina del Trabajo del siglo XXI significa un reto adaptar sus objetivos más clásicos a una nueva población trabajadora. Manteniendo la promoción de la salud, la prevención de enfermedades, y el proporcionar los tratamientos a los trabajadores que sufran patología, debe ir hacia una mayor implicación en las mejoras de la calidad de vida intentado convertir el trabajo en una oportunidad de bienestar. Se tendrá en cuenta todo lo expuesto para hacer de una nueva situación de incertidumbre una oportunidad de innovar y mejorar. En este sentido, podría hacerse lo siguiente:

a) Tener la investigación como estrategia. Los Médicos del Trabajo deben establecer redes de investigación con Centros de referencia como la Escuela Nacional de Medicina del Trabajo, el Instituto Nacional de Seguridad e Higiene en el Trabajo, las Unidades Docentes de Medicina del Trabajo, los Servicios de Prevención de Riesgos laborales, Sociedades de Prevención, Departamentos Universitarios afines (Medicina Legal, Toxicología, ...), etc., etc.. La población a la que atienden ofrece múltiples oportunidades de investigación, de tipo epidemiológico o experimental, en todas las patologías, no olvidemos todo lo relativo con lo cardiovascular, la hipertensión o la diabetes; las diferencias sexuales en la respuesta a agentes tóxicos o a otros estímulo presentes en el medio laboral; otro campo de interés puede ser la objetivación de la influencia de los aspectos psicosociales negativos a través de la investigación de modificaciones en hormonas de estrés u otros indicadores inmunitarios o genéticos y los siempre interesantes trastornos músculo-esqueléticos.

b) Aprovechar la edad laboral desde sus inicios para educar en hábitos saludables, "nunca es demasiado pronto ni demasiado tarde para empezar a cuidarse, pero cuanto antes se haga mejor"; el lograr que las personas incorporen hábitos saludables en sus edades jóvenes ayudará a que mantengan un buen estado de salud hasta edades más altas, lo que proporcionará trabajadores sanos aún en edades próximas a la jubilación (aunque esta se prolongue) y después de la misma.

c) La mayor proporción de población inmigrante en la población trabajadora del futuro. Esto obliga a favorecer su integración en el medio social en el que vivan a través de una adecuada y completa integración laboral; para ello es preciso conocer mejor su marco de procedencia y sus necesidades para la mejor adaptación a su nuevo ambiente.

d) La necesidad de ampliar los objetivos hacia la búsqueda del mayor bienestar posible en el ambiente laboral con estrategias de desterrar en la medida de lo posible el estrés laboral. En los últimos años se ha llamado la atención sobre los riesgos psicosociales caracterizados por ambientes de trabajo traumáticos en lo emocional y afectivo, que acaban ocasionando a los trabajadores patología psicosomática que llega incluso a la incapacidad para el trabajo. Estos cuadros se han concretado en el llamado "burnout" (síndrome de estar quemado) o el "mobing" o acoso laboral, o la mayor frecuencia de cuadros depresivos o ansiosos o los trastornos del sueño, que cuando se prueba que tiene como origen el trabajo han acabado siendo reconocido por los tribunales como AT ya que no se incluyeron, finalmente, como EP, aunque se discutió y valoró esta posibilidad a nivel europeo.

En este sentido, traemos unas reflexiones acerca de lo que debe ser el lugar de trabajo, donde tantas personas pasan una gran parte de su vida.

La Medicina del Trabajo a través de las acciones del Médico del Trabajo como líder del grupo de salud laboral tiene mucha tarea por delante para que el lugar de trabajo o espacio de trabajo y el tiempo del trabajo sea lo más agradable posible para todos los que comparten las tareas y el ambiente diariamente. El ambiente laboral debe constituir un "espacio" que sea para la persona trabajadora prolongación del suyo personal y familiar, que le resulte agradable y le permita encontrar la oportunidad de dar lo mejor de sí misma, en lo laboral y en lo personal. 
El espacio de trabajo debe ser el adecuado en su estructura física, en los medios de que esté dotado y en las personas que lo ocupen. Cualquiera que sea la tarea se hará con mayor eficacia y eficiencia cuando el espacio es agradable y cálido, y genera seguridad y confianza para asumir las responsabilidades cotidianas. Conseguir este espacio depende de quienes tienen las responsabilidades de organización y gestión, aunque todos los que participan en el proceso tienen que poner su propia parte.

Lo contrario a lo que acabamos de referir nos lleva al concepto del "no-espacio", o no-ambiente; es decir, allí donde la persona se ubica para desarrollar su tarea es un lugar de anonimato y de despersonalización.

Hemos tomado esta idea del concepto de no-espacio o no-lugar propuesto por Marc Augé $^{12}$ que describe estos no-lugares asociados a avances y modos de vida contemporáneos, enclaves anónimos para hombres y mujeres anónimos, que deambulan como autómatas. Dice el autor: "Si un lugar puede definirse como lugar de identidad, relacional e histórico, un espacio que no puede definirse como espacio de identidad ni como relacional ni como bistórico, definirá un no-lugar. La hipótesis aquí defendida es que la sobremodernidad ${ }^{8}$ esproductora de no-lugares, es decir, de espacios que no son en sílugares antropológicos...".

Para Augé los no-lugares o no-espacios de nuestra sociedad moderna son espacios físicos, ocupados por personas en las que la comunicación está ausente y la palabra pierde su contenido sentimental y emotivo para hacerse vehículo frío de intereses materiales, las miradas se tornan furtivas $\mathrm{u}$ hostiles entre personas en constante desencuentro, todo ello opuesto al espíritu y a la dimensión trascendente de la persona.

Llevado esto a los ambientes de trabajo, encontramos que la persona trabajadora puede toparse de lleno en la realización de su actividad con el "no-espacio", "no-lugar" o "no-ambiente". Sería un lugar en el que puede contarse con recursos adecuados, tecnología punta y otros medios, pero en el que llegar a estar ausente el respeto, la cordialidad, los buenos modos; sería el espacio dominado por el desapego, el trato desatento y hasta el desprecio y el maltrato psíquico. Se puede comprender fácilmente que este "no-espacio" acabe afectando a la persona en su salud integral. Por ello es función de la Medicina del Trabajo del siglo XXI combatir este aspecto de las relaciones interpersonales de los trabajadores.

La humanización del ambiente del trabajo debe ser tarea principal en salud laboral. Tengamos en cuenta, para revertirla, la frase de Miguel Delibes "la máquina ha venido a calentar el estómago del hombre, pero ha enfriado su corazón”.

El envejecimiento activo debe ser aprovechado desde una perspectiva positiva, manteniendo a las personas en su ámbito laboral en la medida que sean capaces y lo deseen. La promoción de la salud desde las edades más jóvenes en el ámbito laboral traerá beneficios para la propia persona a lo largo de toda su vida, pero también para la sociedad en general, no sólo en lo económico. En todo ello tiene la Medicina del Trabajo un papel de suma importancia para el que estamos preparados.

\section{BIBLIOGRAFÍA}

1. Bernardino Ramazzini De morbis artificum diatriba. Padova, 1713.

2. Bernardino Ramazzini. Tratado de las Enfermedades de los artesanos. Reedición de 2003 del Ministerio de Sanidad, Instituto de Salud Carlos III y la Escuela Nacional de Medicina del Trabajo, de la Edición de 1983. Traducción y notas de José Luís Moraleja y Francisco Pejenaute. Estudio preliminar de Francisco L. Redondo. Madrid 1983.

3. Reverte Coma, J.M. Bernardino Ramazzini y su obra. En Medicina e Historia. Editores: Agustín Albarracín Teulón, José M. ${ }^{a}$ López Piñero y Luís Granjel. Editorial de la Universidad Complutense de Madrid. 1980.

4. World Population Proyects. United Nations of Economic and Social Affaire. Population Division. 2014.

8 Este concepto encaja en el "postmodernismo", comentado en este artículo como situación de pérdida de implicación personal y emocional en los acontecimientos sociales en todas sus manifestaciones. 
5. Castellano Arroyo M. ${ }^{a}$ Valoración médico-forense del agresor. En Violencia de Género: Valoración médicoforense y jurídico-penal. Coord: M. ${ }^{a}$ Sol Rodríguez Calvo. Editorial Tirant lo Blanch. Valencia, 2013.

6. Gans E. Chonicles of love and resentment. University of California. Los Ángeles, 2000.

7. Requena M. Estratificación y clases sociales en España. Capítulo V. Informe España 2011. Fundación Encuentro.

8. Garrido Medina L. Demografía longitudinal de la ocupación. Información Comercial Española, ICE; Revista de Economía, ISSN 0019-977X, n. ${ }^{\circ}$ 815, pp 105-142.

9. Cien años de Medicina en España. Actualidad Médica. Suplemento II 792. 2014. ISSN: 0365-7965 (www. actualidadmedica.es).

10. Durán F. Informe sobre Riesgos laborales y su prevención. Madrid, 2000.

11. Castellano Arroyo $M^{a}$. La Medicina del Trabajo en la España del siglo XXI. ¿Cambiar para progresar? La

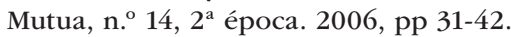

12. Augé M. Los no lugares: Espacios del anonimato. Editorial Gedisa. Barcelona 1993.

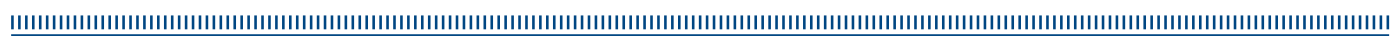

